\title{
Conditions for effective Nd-YAG laser angioplasty
}

\author{
HERBERT J GESCHWIND, GEORGES BOUSSIGNAC, BERNARD TEISSEIRE, \\ NICOLE BENHAIEM, RENEE BITTOUN, DANIEL LAURENT
}

\begin{abstract}
From the Departments of Clinical Investigation and Histopathology, University Hospital Henri-Mondor, Créteil, France
\end{abstract}

SUMMARY To establish the optimal conditions for recanalisation of obstructed arteries without damage to vessel walls, a Nd-YAG laser coupled to a $0.2 \mathrm{~mm}$ diameter optic fibre was used on obstructed human cadaver coronary and peripheral arteries and on popliteal arteries in amputated limbs. Vaporisation of atheromatous plaques was consistently obtained with an energy of 360-600 J and a diluted blood perfusate $(3 \mathrm{~g} / 100 \mathrm{ml}$ haemoglobin) at a rate of $20 \mathrm{ml} / \mathrm{min}$. The arterial wall was protected from thermal injury by inserting the optic fibre into an inflated balloon catheter and by cooling the system with the perfusate. Since recanalisation of occluded arteries was consistently obtained without damage to the arterial wall or debris and thin and flexible optic fibres were easy to guide in the arteries, perutaneous transluminal Nd-YAG laser angioplasty was used in obstructed femoral and popliteal arteries in three patients.

The first European trials in man showed the method to be feasible, effective, and harmless, although further studies are required to improve penetration of the obstruction and increase the diameter of tunnel.

Lasers have been widely used in the treatment of diseases in several areas of medical practice. This technique has not, however, yet been applied in diseases of the heart and blood vessels. Experimental studies have recently been undertaken to assess the efficacy of laser radiation for removing obstructions in test tubes, ${ }^{1}$ in human cadaver arteries, ${ }^{2}$ or in animal arteries. ${ }^{3}$ The obstructions were either thrombi or atheromatous plaques. The sources of laser that have been used comprised carbon dioxide, Nd-YAG (neodymium yttrium, aluminium, garnet), and mainly argon. ${ }^{4}$ These studies concluded that atheromatous plaques and thrombi may be recanalised, but damage to the arterial wall often occurred.

Since the optimum conditions for successfully perforating obstructed arteries and avoiding injury to the arterial wall have not yet been determined, we carried out experimental studies with a Nd-YAG laser coupled to a fibreoptic system to establish the main suitable conditions required to relieve obstructions in arteries without perforating the arterial wall..$^{5}$

Requests for reprints to Dr Herbert J Geschwind, CHU Henri Mondor, 51 avenue du Maréchal de Lattre de Tassigny, 94010 Créteil, France.

Accepted for publication 2 August 1984

\section{Material and methods}

Experimental studies were performed on various material including: (a) 10 thrombi prepared from blood samples from normal human volunteers; the blood was permitted to clot and dry in test tubes of $5 \mathrm{~mm}$ in diameter, and the thrombi were cut to fixed lengths of $5 \mathrm{~mm}$; (b) 10 atheromatous plaques removed from obstructed fresh human cadaver arteries and inserted into test tubes; (c) 20 totally occluded fresh human cadaver coronary arteries cut to lengths of $5 \mathrm{~mm}$; and (d) 10 totally occluded popliteal and tibial arteries located in freshly amputated limbs.

A Nd-YAG laser with a wavelength of $1064 \mathrm{~nm}$ (Robert et Carrière Biomédical) was coupled to a fibreoptic system consisting of a $0.2 \mathrm{~mm}$ diameter silica fibre $3 \mathrm{~mm}$ long. One end was aligned with the focused laser beam as it exited from the laser, and the free end was used for laser emission. The power reading on the laser meter was 20-25 W and the actual power at the fibretip $12 \mathrm{~W}$. The main laser beam was used with continuous emission, and duration of exposure varied between 2 and $50 \mathrm{~s}$. Peak emission was also used and the duration adjusted by an electronic system to $0.2,0.3,0.5$, and $0.7 \mathrm{~s}$ with automatic firing in bursts at a rate of one emission every $5 \mathrm{~s}$. The 
divergence at the tip of the optic fibre was $10^{\circ}$. In order accurately to locate the impact area from the main beam a low power red beam (having no effect on tissues) was emitted when desired by an $\mathrm{He}-\mathrm{Ne}$ laser, thus showing the beam trajectory.

The optic fibre was inserted into a balloon catheter and both were advanced against the atherosclerotic plaque or the thrombus. The balloon was inflated in order to maintain the coaxial position of the optic fibre. For laser treatment of coronary arteries, the device commonly used in coronary angioplasty procedures was used. Both the optic fibre and the balloon catheter were inserted into a guiding catheter so that the tip of the optic fibre was placed $3 \mathrm{~mm}$ beyond the tip of the balloon catheter. When test tubes were used the laser beam was directed through the open end.

During the procedure fluid was circulated in both the guiding and balloon catheters using a Transomega T4 pump. Experiments were achieved using: (a) blood (haemoglobin $15 \mathrm{~g} / \mathrm{dl}$ ); (b) saline; and (c) a mixture of blood and saline so that the haemoglobin concentration was $3 \mathrm{~g} / \mathrm{dl}$. The rate of perfusion was adjusted to 10,20 , and $50 \mathrm{ml} / \mathrm{min}$. Blood was filtered from the artery effluent through 8 and $3 \mu \mathrm{m}$ micropore filters.

The effects of laser emission were evaluated by gross inspection and microscopically; those in obstructed amputated limb arteries were assessed by repeat angiography. In intact occluded coronary artery segments the treatment was considered to be successful if the plaque was penetrated and a new lumen established. The diameter of holes was measured using a stereoscopic microscope (Wild Heerbrugg M8). Perforation was defined as a rupture through the entire thickness of the arterial wall.

After exposure to the laser, all tissue samples were fixed in $10 \%$ formaldehyde, dehydrated, and embedded in paraffin. Tissue blocks were cut in sections of $3 \mu \mathrm{m}$ thickness. Sections from each tissue were stained with haematoxylin and eosin, Masson's trichrome, and orcein. Each section was evaluated by light microscopy.

The total energy delivered in joules was calculated as the product of the amount of power (watts) and the duration of exposure (s).

\section{Results}

\section{EFFECTS ON THROMBI}

Retraction of thrombi occurred in all cases, and a reduction in size by $50-70 \%$ was obtained with $200 \mathrm{~J}$. Totally obstructive thrombi were detached from the walls of test tubes and arterial walls, and holes and perforations were created.

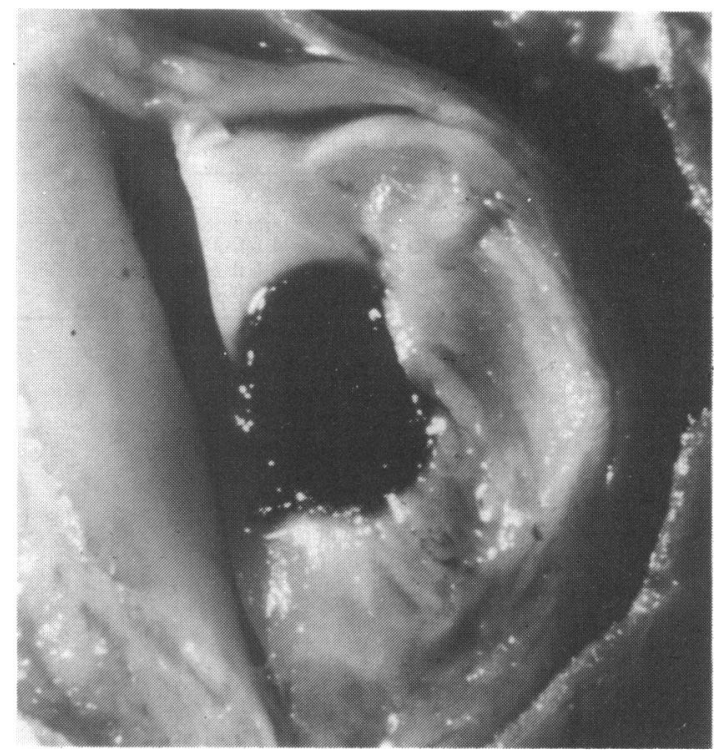

Fig. 1 Hole obtained with Nd-YAG laser emission in an obstructed fresh human cadaver carotid artery viewed through a stereoscopic microscope.

\section{EFFECT ON ATHEROMATOUS PLAQUES}

The effects were dependent on the colour, thickness, density of tissue, ${ }^{6}$ power intensity, and duration of exposure to the laser as well as the composition and rate of the perfusion. With saline, no significant effects were observed. With blood, thermal injury occurred at the entry of the plaque but no perforation resulted. By contrast, with diluted blood $(3 \mathrm{~g} / 100 \mathrm{ml}$ haemoglobin concentration) perfusion, perforation of the atheromatous plaque occurred (Fig. 1). The most efficient perfusion rate was $20 \mathrm{ml} / \mathrm{min}$ with a perfusate temperature of $22^{\circ} \mathrm{C}$ (Tables 1 and 2). Under these

Table 1 Results of laser treatment on atheromatous plaques according to the perforate used. Values are mean $(S D)(n=8)$

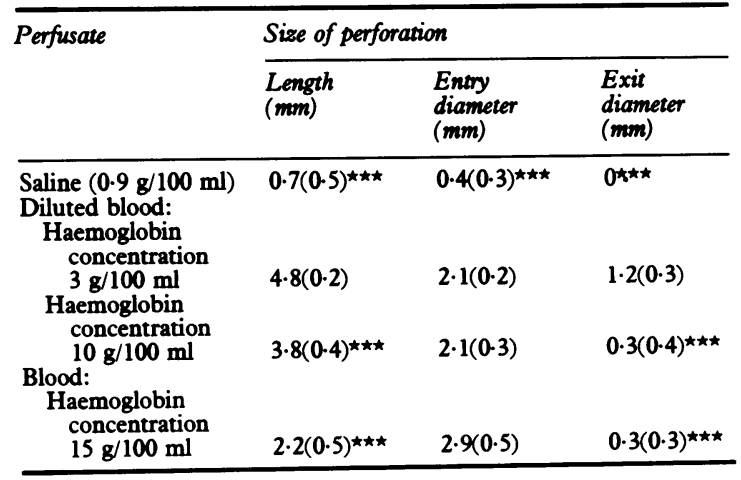

$\star \star \star \mathrm{p}<0.001$. 
Table 2 Results of laser treatment on atheromatous plaques according to temperature of perfusate. Values are mean $(S D)(n=8)$

\begin{tabular}{llll}
\hline Size of perforation & \multicolumn{4}{l}{ Temperature } \\
\cline { 2 - 4 } & $4^{\circ} \mathrm{C}$ & $22^{\circ} \mathrm{C}$ & $37^{\circ} \mathrm{C}$ \\
\hline Length (mm) & $4 \cdot 6(0.4)$ & $4 \cdot 8(0.2)$ & $4 \cdot 8(0.2)$ \\
Entry diameter (mm) & $1 \cdot 9(0.4)$ & $2 \cdot 1(0.2)$ & $2 \cdot 0(0.3)$ \\
Exit diameter (mm) & $1 \cdot 0(0.4)$ & $1 \cdot 2(0.3)$ & $1 \cdot 2(0.2)$ \\
\hline
\end{tabular}

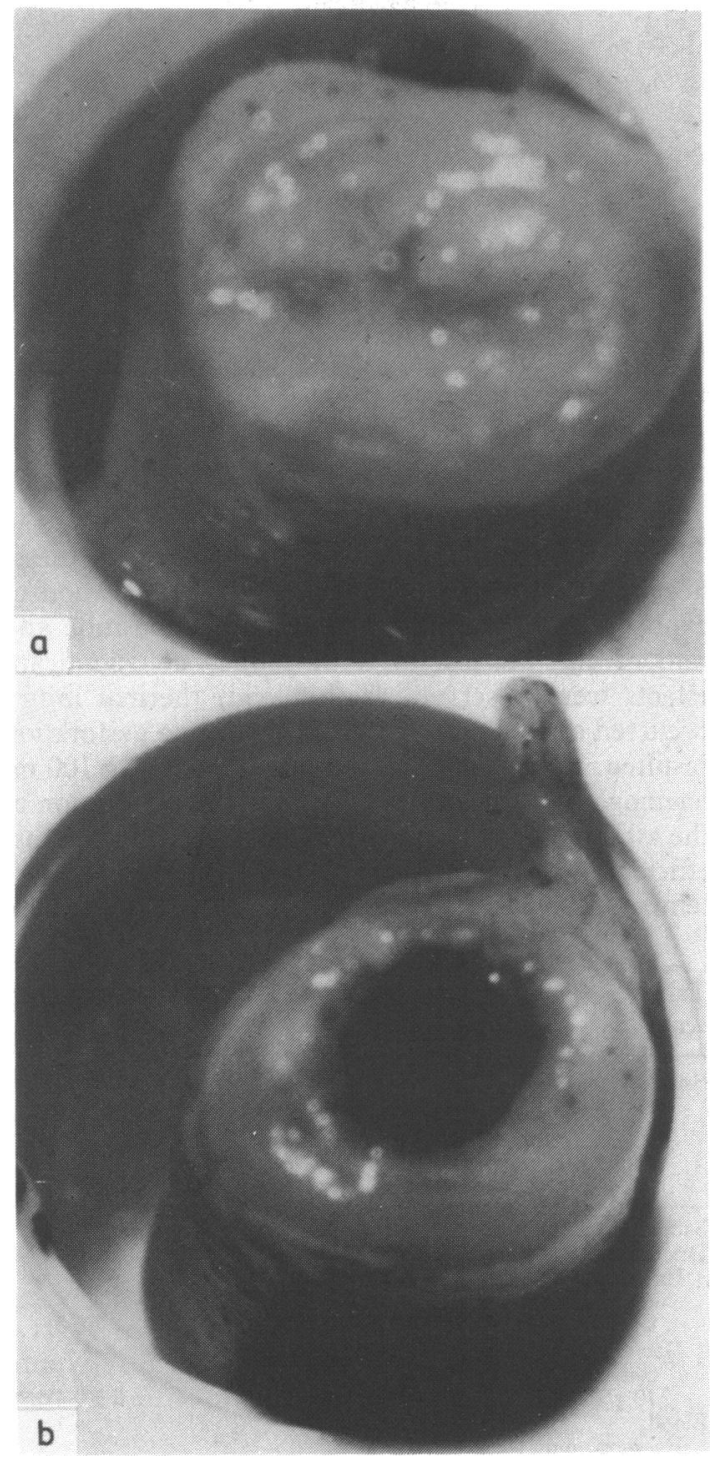

Fig. 2 An obstructed fresh human cadaver coromary artery (a) before laser treatment and (b) after penetration with a laser beam (energy $360 \mathrm{f}$; energy density $36000 \mathrm{f} / \mathrm{cm}^{2}$ ). experimental conditions the energy required to penetrate a $5 \mathrm{~mm}$ long plaque was $12 \mathrm{~W}$ at the tip of the optic fibre and the exposure duration $30 \mathrm{~s}$, the energy density being $36000 \mathrm{~J} / \mathrm{cm}^{2}$ (Fig. 2). Three firings of $480 \mathrm{~J}$ each were necessary totally to penetrate plaques $20 \mathrm{~mm}$ long. The diameter of the hole at the entry of the tunnel was $2 \mathrm{~mm}$ and $1 \mathrm{~mm}$ at the other end. No effect could be obtained when laser emission was used in bursts. Five centimetres of occluded amputated limb arteries were cleared with nine successive firings of $600 \mathrm{~J}$ each.

\section{EFFECT ON THE ARTERIAL WALL}

Only three perforations of the arterial wall occurred. In these cases the optic fibre had been inserted into the arterial lumen without maintaining coaxial position with the inflated balloon catheter. When the coaxial position was maintained no damage to the arterial wall occurred, and no leak of contrast medium was seen at repeat angiography during recanalisation of the amputated limb arteries (Fig. 3).

Neither clots nor debris appeared to be retained on the micropore filter from the perfusion effluent after laser exposure.

\section{HISTOLOGICAL FINDINGS}

No matter which haemoglobin concentration was used, histological examination showed thermal injury, fissures, retraction, and dissection of the atherosclerotic plaques. With a saline perfusion, the fracture of the lesion was insufficient to create a viable pathway. Using the $15 \mathrm{~g} / \mathrm{dl}$ haemoglobin concentration the vascular wall thickness suffered thermal injury, but no perforation occurred. No perforation of the vascular wall occurred with saline and the $3 \mathrm{~g} / \mathrm{dl}$ haemoglobin perfusate but only slight thermal injury to the wall.

\section{Discussion}

Laser energy carried by optic fibres appears to be an efficient method of improving perfusion through obstructed arteries. This was shown by perforations consistently obtained on atherosclerotic plaques or thrombi.

The diameter of the holes at the entry of the tunnel and at the other end were large enough appreciably to improve arterial perfusion. This was demonstrated by control angiograms, which showed satisfactory penetration of obstructed popliteal and tibial arteries from amputed limbs.

Maximum efficacy was observed when lasing was used with a diluted blood perfusion of $3 \mathrm{~g} / \mathrm{dl}$ haemoglobin concentration. This may be due to the fact that with saline the laser beam was not absorbed by the white and yellow coloured plaque. With blood, the absorption of the laser beam was likely to be too high 


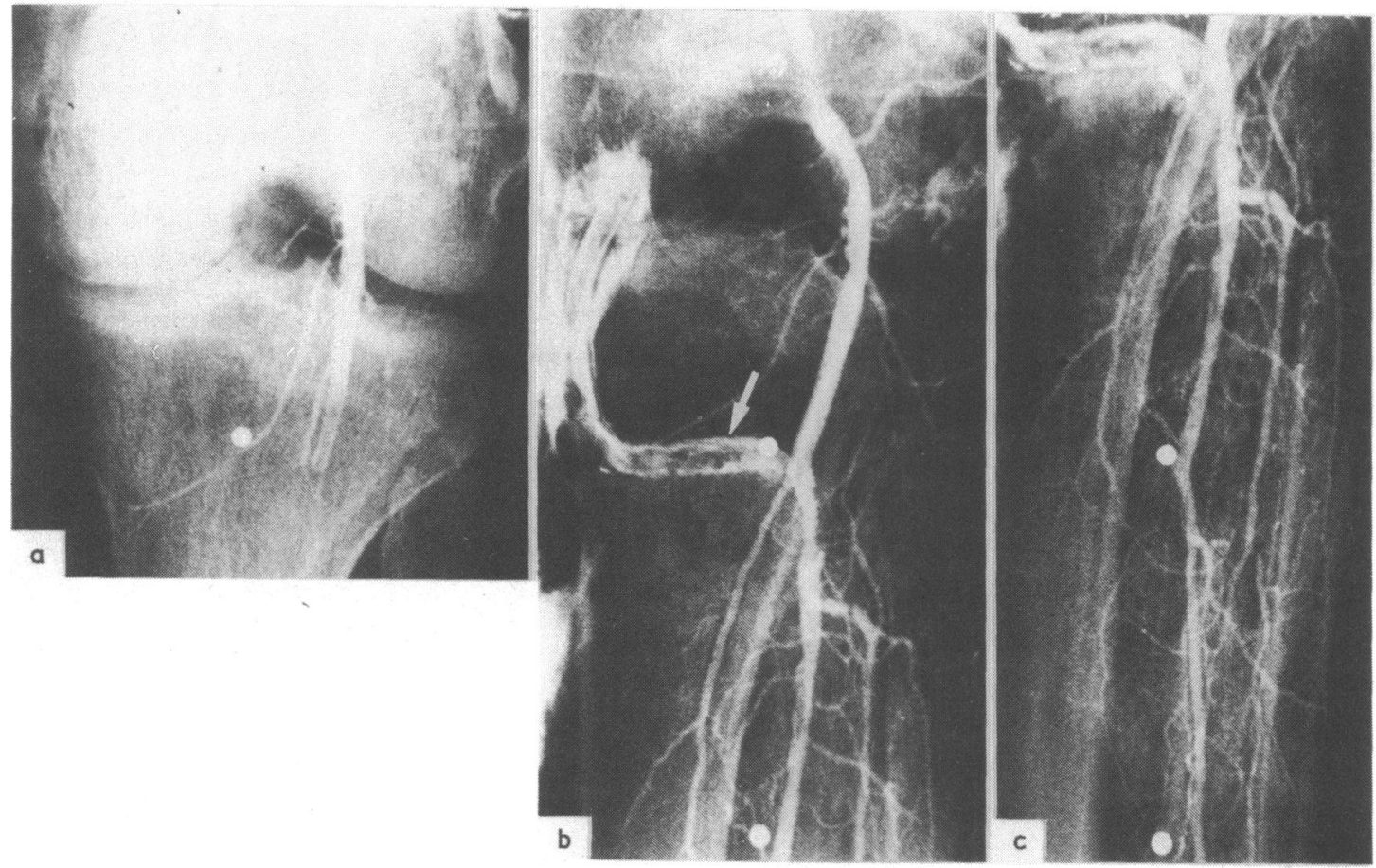

Fig. 3 Angiograms of (a) an obstructed popliteal artery in a freshly amputated limb; (b) recanalisation of popliteal and tibial arteries after laser treatment; note retrograde opacification of a previous obstructed bypass (arrow); (c) repermeation of distal vessels in the same limb.

at the entry of the plaque, thus preventing the beam from penetrating into the atheroma. With diluted blood, the absorption of the laser beam was sufficient to allow perforation to occur and the plaque to be entirely penetrated, since more residual energy was available.

The rate of perfusion also played a role in the efficacy of vaporising the atheroma, since a high rate of perfusion prevented thermal injury. By contrast, a low rate of perfusion permitted blood to absorb the laser radiation to too great an extent. Consequently, insufficient laser energy was available and thermal injury to the plaque did not occur.

An adequate rate of perfusion was required to prevent the arterial wall from being injured by the laser beam since the circulating fluid acts by cooling the artery. The arterial wall was protected by the coaxial position of the tip of the optic fibre in the centre of the arterial lumen since the inflated balloon kept the fibre tip at a distance from the arterial wall.

Laser treatment was effective only with continuous emission when the duration was long enough to create thermal injury to the obstruction. By contrast, when bursts were used cooling occurred during the emission intervals leading to a ineffective response.
The safety of the procedure was shown by the fact that no debris was retained on the micropore filters; thus no distal embolisation is likely to occur. This agrees with the findings of Choy et al who observed total vaporisation without debris then thrombi were subjected to laser emission. ${ }^{3}$

Finally, since the optic fibre that was used in our experiments was very thin and flexible it could be inserted into all available balloon catheters including those commonly used for peripheral artery angioplasty and specifically those used for coronary angioplasty. The feasibility of guiding these devices in man was evident both in the femoral and iliac arteries as well as in the coronary arteries.

\section{CLINICAL STUDY}

Since these experimental studies consistently showed effective recanalisation of totally occluded arteries without perforation of the arterial wall the method was recently applied in three patients. The protocol was approved by the human research committee of this hospital and informed consent was obtained from all patients. The first patient had a totally occluded femoral artery, the second a totally occluded popliteal artery, and the third a femoral artery stenosis $5 \mathrm{~mm}$ 

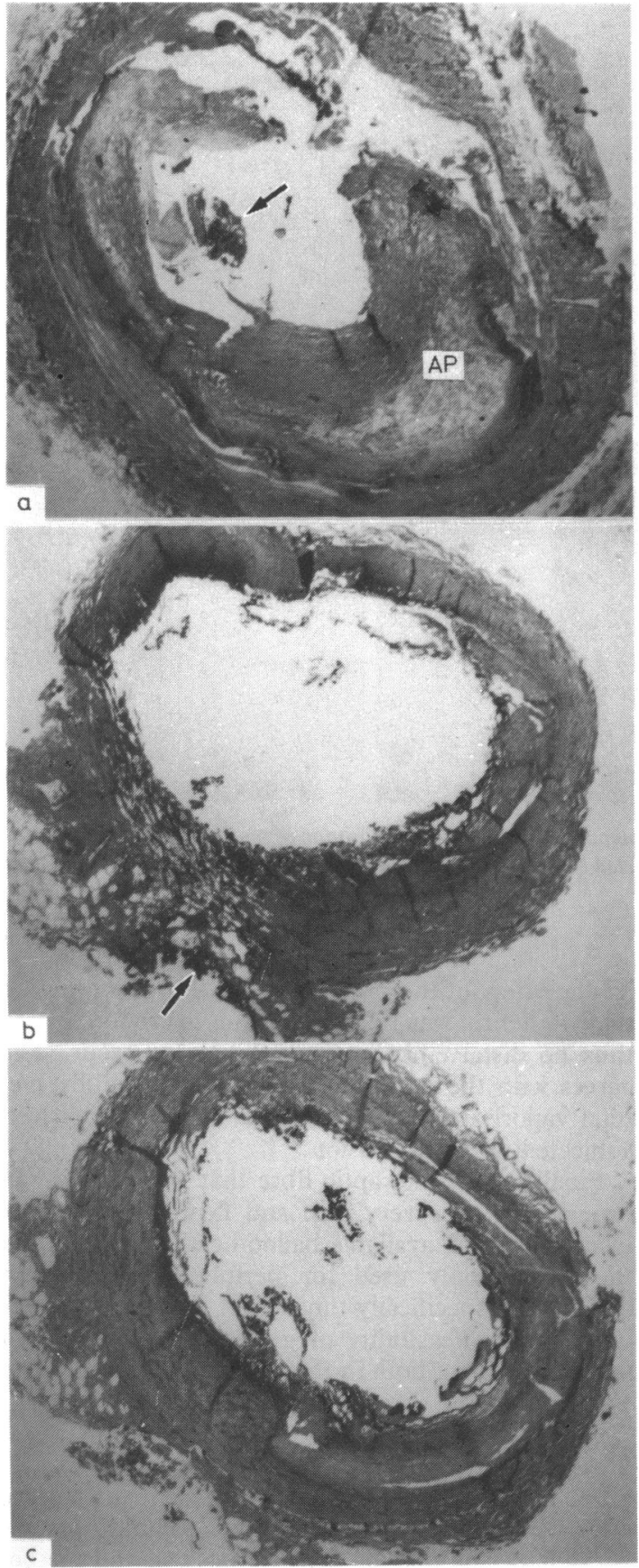

Fig. 4 Histological specimens showing the effects of laser treatment. (a) In an atheromatous vascular wall treated using a saline perfusate the atherosclerotic plaque $(A P)$ is lightly penetrated. Note the thermal injury (arrow). Masson's trichrome stain $\times 32$. (b) Treatment with the $15 \mathrm{~g} / 100 \mathrm{ml}$ haemoglobin perfusate produced thermal necrotic debris in the adventitia (arrow). Mainly necrotic intimal and medial cells can be seen. Masson's trichrome stain $\times 32$. (c) Treatment with $3 \mathrm{~g} / 100 \mathrm{ml}$ haemoglobin perfusate produced efficient recanalisation of the occluded arterial lumen without major injury to the wall. Masson's trichrome stain $\times 32$.
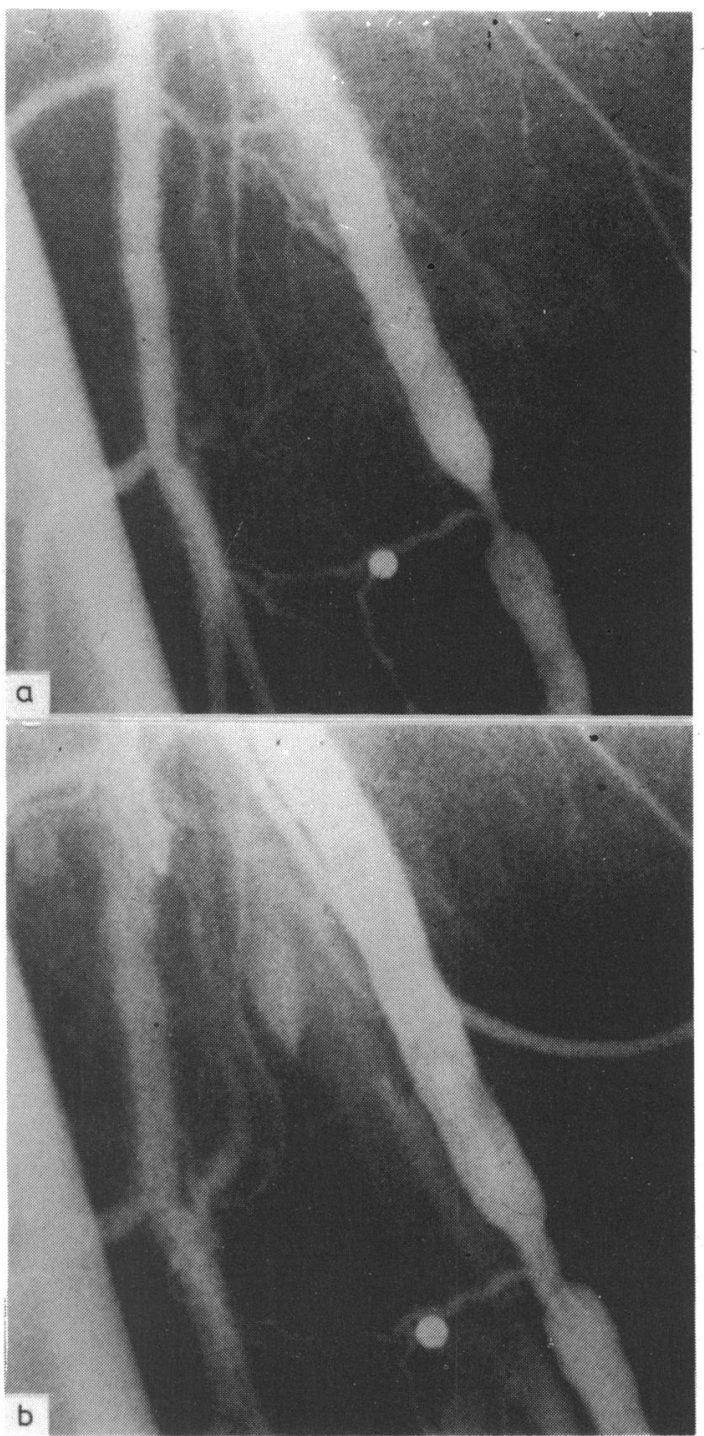

Fig. 5 Angiograms of femoral artery stenosis in man (a) before and (b) after enlargement of the stenosis by the laser. 
long and $1 \mathrm{~mm}$ in diameter. The balloon catheter and the optical fibre were inserted percutaneously into the femoral artery. Three firings of $600 \mathrm{~J}$ each were necessary to recanalise the occluded femoral and popliteal arteries. Three firings of $360 \mathrm{~J}$ each were required to enlarge the femoral artery stenosis from 1 to $4 \mathrm{~mm}$ (Fig. 5). No perforation of the arterial wall occurred. The only side effect was a burning sensation in the limb during laser emission.

These clinical trials of laser recanalisation in obstructed peripheral arteries are to our knowledge the first in Europe to be carried out in man. To date, the method appears to be limited as the stenosis was not entirely relieved; only a narrow tunnel could be obtained in the totally occluded arteries and follow up was not possible. Although further studies are required to improve penetration of the obstruction and increase the diameter of the tunnels, these cases show that percutaneous transluminal laser angioplasty is feasible, effective, and harmless in man.

We thank B Gaujour, O Legoff, and P Mayiolini for their technical assistance.

The research was supported by grants from the
Institut de la Santé et de la Recherche Médicale (INSERM), Research Unit U138, and the French Ministry of Research and Industry.

\section{References}

1 Lee G, Ikeda RM, Stobbe D, et al. Effects of laser irradiation on human thrombus: demonstration of a linear dissolution-dose relation between clot length and energy density. Am f Cardiol 1983; 52: 876-7.

2 Choy DSJ, Stertzer SH, Rotterdam HZ, Bruno MS. Laser coronary angioplasty: experience with 9 cadaver hearts. Am $\mathcal{F}$ Cardiol 1982; 50: 1209-11.

3 Choy DSJ, Stertzer S, Rotterdam HZ, Sharrock N, Kaminow IP. Transluminal laser catheter angioplasty. Am F Cardiol 1982; 50: 1206-8.

4 Abela GS, Normann S, Cohen D, Feldman RL, Geiser EA, Conti CR. Effects of carbon dioxide, Nd-YAG, and Argon laser radiation on coronary atheromatous plaques. Am f Cardiol 1982; 50: 1199-205.

5 Geschwind H, Boussignac G, Teisseire B, et al. Laser angioplasty: effects on coronary artery stenosis [Letter]. Lancet 1983; ii: 1134.

6 Lee G, Ikeda R, Herman I, et al. The qualitative effects of laser irradiation on human arteriosclerotic disease. Am Heart f 1983; 105: 885-9. 